3. In der hypertrophierten Muskelwand treten neben normalen Zellverbänden Bezirke mit degenerativen Zellveränderungen auf. Diese betreffen das Muskelgewebe ebenso wie die Innervation. Die Veränderungen lassen sich deutlich am Zustandsbild der Zellen und ihrer Kerne demonstrieren. Muskelzellen und Ganglienzellen des Auerbachschen Plexus weisen gleichartige Abweichungen in der gesamten Antrumwand auf. Wir zeigen: Cytoplasma und Kernvakuolisierung-, Karyolyse-, Karyorrhexis und Kernpyknose.

Beim Ulcus ventriculi liegen somit in der Muskelwand des gesamten Canalis egestorius degenerative Gewebeveränderungen vor.

\title{
6. Spleno-pulmonaler Shunt mit autologem Venentransplantat beim Hund
}

\author{
H. H. Gruenagel, B. Domres, L. Neumann und V. Weber \\ Chirurgische Abt. d. Ev. Krankenhauses Düsseldorf \\ und Chirurgische Universitätsklinik Tübingen
}

Es wird eine Methode zur direkten Ableitung des Pfortaderblutes über die Milzvene in die Lungenunterlappenvene unter Zwischenschaltung eines autologen Venentransplantats beim Hund angegeben. Die Anastomosen an Milz- und Lungenvene wurden mit der Ringmethode nach Kunlin vorgenommen und das Transplantat am Zwerchfelldurchtritt mit einem Celestintubus geschient. Die Versuchsanordnung ermöglicht die Abklärung kreislaufdynamischer und stoffwechselbedingter Vorgänge bei der Einleitung des Pfortaderblutes in den großen Kreislauf unter Umgehung des rechten Herzens und der Lungenpassage. Intraoperative Strömungsmessungen (Nycotron, Siemens-AG) ergaben eine Ableitung von mehr als zwei Drittel des Pfortaderströmungsvolumens über das Venentransplantat in die Lungenvene nach Verschluß der Pfortader am Leberhilus. Der Pfortaderdruck ist dabei geringgradig erhöht. Therapeutische Indikationen eines solchen Vorgehens sind Formen des BuddChiari-Syndroms und eine Aplasie der Vena cava inferior mit portaler Hypertension und blutenden Oesophagusvaricen.

Eine Schemazeichnung und 5 Abbildungen über die Operationsanordnung und 1 Abbildung postoperativer Röntgenangiographie über die Abflußverhältnisse des Pfortadersystems nach Spleno-pulmonaler Anastomose. 